\title{
Dentistry
}

\section{Axiographic Assessment of Masticatory System function in Healthy Volunteers}

\author{
Wojciech Kondrat ${ }^{1}$, Teresa Sierpinska ${ }^{2 *}$ and John Radke ${ }^{3}$ \\ ${ }^{1}$ Department of Prosthetic Dentistry, Medical University of Bialystok, Poland \\ ${ }^{2}$ Department of Dental Techniques, Medical University of Bialystok, Poland
}

${ }^{3}$ Bioresearch Associates, USA

\section{Abstract}

Background: The use of axiographic devices provides a simple and minimally invasive way to detect masticatory abnormalities, enabling early preventive action and treatment with a long-term impact on the good functioning of the masticatory system

Objective: The aim of the study was to collect normative data from the stomatognathicsystem using elecrtonic axiography and to find the differences between males and females.

Material: The study evaluated 98 females and 88 males, in a mean age of 19 years with full natural dentition corresponding to Angle's Class I.

Methods: All the participants undertook clinical examination and electronic axiography using the Cadiax Compact II.

Results: A significantly higher Quantity Maximal S3d in men $(p=0.021)$, a trend towards higher Transverse Condyle Inclination Values $3 \mathrm{~mm}$ in women and a significantly lower reproducibility of the opening and closing movements were found in women $(p=0.022)$.

Conclusion: The graphs of opening and closing movements in a group of healthy women and men with no symptoms from the masticatory system differed significantly between genders.

The Quantity Maximal S3d parameter is higher in men, but because of the large range of values, this parameter cannot be a single criterion used to detect TMJ dysfunction.

Keywords: Computerized axiography; Masticatory system; Diagnostics

\section{Introduction}

Most patients reporting to a dental office at periodic check-ups have no symptoms of masticatory system dysfunction. A carefully conducted clinical examination is critically important, but does not allow for detection of all abnormalities. McNeill [1] states that approximately $75 \%$ of the population may experience one of the many signs of masticatory dysfunction. Other authors also recognize the frequent occurrence of masticatory system dysfunction even in very young people $[2,3]$. In order to detect existing anomalies in the masticatory organ, it is necessary to have broad clinical experience. Often, the early stages of dysfunction are missed during diagnosis. Mainly, they are subluxation, without associated symptoms of pain or dysfunction, or the increased mobility of the articular disc. Usually they remain undiagnosed and untreated [4-15]. Modern axiographic devices are very helpful in assessing the proper functioning of the masticatory system [16-21]. They can record the movement of the rotation point of the hinge axis of the mandible in three dimensions and they are used to record the movements of the temporomandibular joint [22-24]. They allow us to make individual measurements of the range of motion of the jaw, and evaluate parameters of its movements. Graphical methods for recording the movements of the jaw allow the measurement of parameters, such as the angle of inclination of the articular path and the Bennett angle [25]. Graphic depiction of mandibular movements provides information for the assessment of temporomandibular joint function [26,27]. Graphs can illustrate the quality of TMJ function, especially if they are done with respect to the repeatable hinge axis [24,28]. Increasingly, attempts have been made to detect any correlations between the graphs of jaw movements and the clinical symptoms of functional disorders. The use of axiographic devices provides a simple and minimally invasive way to detect masticatory abnormalities, enabling early preventive action and treatment with a long-term impact on the good functioning of the masticatory system [29].

Considering that young healthy adults with full natural dentition usually did not report any complaints from temporo mandibular joints the main purpouse of the study was to collect normative data from the stomatognathic system using elecrtonic axiography and to find the differences between males and females.

\section{Material and Methods}

The study was conducted in a group of young people attending secondary schools and students of the first year of college studies. The information regarding the research was presented to the governments and parents of five high schools, so around 1500 young persons were informed and taken into consideration when planning the research. For the sake of the participation to the study was voluntary and taking into consideration inclusion/exclusion criteria, finally, the study involved 186 people- 98 females and 88 males, aged 18-21 years - mean age of 19 years.

*Corresponding author: Teresa Sierpinska, PhD, Department of Dental Techniques, Medical University of Bialystok Waszyngton Str.13, 15-276 Bialystok, Poland, Tel: 04885 7468349; Fax 048857447030; E-mail: teresasierpinska@gmail.com

Received July 14, 2018; Accepted July 30, 2018; Published August 04, 2018

Citation: Kondrat W, Sierpinska T, Radke J (2018) Axiographic Assessment of Masticatory System function in Healthy Volunteers. Dentistry 8: 504 doi:10.4172/2161-1122.1000504

Copyright: ( 2018 Kondrat W, et al. This is an open-access article distributed under the terms of the Creative Commons Attribution License, which permits unrestricted use, distribution, and reproduction in any medium, provided the original author and source are credited. 
The subjects were required to meet the following criteria for the inclusion in the study:

Class 1 molar and canine relations;

1. Full natural dentition with well-aligned arches;

2. Well-related vertical, transverse, and anteroposterior relationships;

3. Normal growth and good health.

\section{Exclusion criteria}

The subjects were excluded from the study when the following criteria were met:

1. Previous orthodontic treatment;

2. Extensive fillings or edentulous spaces;

3. History of trauma in the region of the masticatory system;

4. Any pain treatment in any region of the masticatory system;

5. Prosthetic treatment before recruitment to the study.

6. Presence of any systemic disease

Data were collected in the Department of Prosthetic Dentistry and the protocol conformed to the criteria of The Helsinki Declaration, ICH Guideline for Good Clinical Practice, and approved by the Local Ethical Committee with an approval number of KBET/89B/2009. The participants were recruited into the study after obtaining consent from educational authorities, school headmasters, parents, and participants themselves.

\section{Study design}

The study included the following:

1. Clinical examination of the masticatory system

2. Muscle deprogramming

3. Instrumental diagnostics

\section{Clinical examination}

The clinical examination took into account the inclusion criteria of joint disorders by Dworkin and Leresche [30]. The study plan consisted of patient history responses and clinical findings according to Diagnostic Criteria for Temporomandibular Disorders for Clinical Application (DC/TMD) [31]. The study card consisted of four parts: personal data, general medical history, specialist history and basic clinical examination. General medical history contained questions about the health of the subjects The specialist history concerned dental diseases, in particular any difficulty chewing, speaking, obtaining proper occlusion of the teeth, hypersensitivity of the teeth, pain or acoustic phenomena in the temporomandibular joints when opening, incising or yawning, the presence of headaches and/or bad posture. The clinical examination included enhanced focus on the muscles and temporomandibular joints.

\section{Muscle deprogramming}

Before starting the instrumental diagnostics patients were subjected to muscle deprogramming. It consisted of repeated opening and closing of the mouth and biting lignin rollers for 10 minutes. Deprogramming was to eliminate the influence of muscle function on the registration, as instrumental analysis was to be measured from habitual movements of the jaw.

\section{Study conditions of instrumental diagnostics}

The subjects underwent three consecutive instrumental recordings of the opening and closing movements at intervals of a few minutes. The examinations were performed always on the same days of the week (Tuesday, Wednesday), and at the same time (between 8.00 AM and $12.00 \mathrm{AM})$. They were carried out under the same conditions and all conducted by one person.

\section{Instrumental diagnostics}

Axiography of the mandible was carried out using the Cadiax Compact II (Gamma Dental, Austria) [22]. It allows for an accurate determination of the path of the moving condyles within the TMJs in the form of graphs displayed in 3 planes (frontal, sagittal and horizontal). The system records the hinge axis of the joints, around which the sagittal rotational movement takes place. An integral part of the device is a facial arch, whose main task is to register the geometric relationship between the axis of rotation of the condyles traced by the reference plane. The arbitrary points of the fixed hinge axis of the temporomandibular joints are transferred through the use of anatomical relationship between the opening of the external ear canal and the head of the mandible. The study using Cadiax Compact II was performed in a comfortable sitting position of the patient. The study applied a standard bite plate attached to the lower arch using hard silicone compound. All movements of the lower jaw were made from the reference position, which was manually marked by the doctor before registration. It was the starting point of recording and was the most repeatable position of the articular condyles.

\section{Statistical Analysis}

Statistical analysis tested the arithmetic mean of three consecutive measurements. The values of the following parameters obtained in the axiographic study were used to assess abduction and adduction of the mandible: Quantity Max. S3D (path of condylar movement from the reference position to the maximum range of movement- in millimetres) $[26,32,33] \cdot$ Reproducibility (value calculated based on the free space between the paths of movement) [34,35].

SCI - Sagittal Condyle Inclination (angle between the sagittal plane and the path of abduction movement measured on the distance of 3 and $5 \mathrm{~mm})[21,32,36]$.

TCI - Transverse Condyle Inclination (angle between the horizontal plane and the path of abduction movement measured on the distance of 3 and $5 \mathrm{~mm}$ ).

The results are shown using numeric tables together with percentage rates. The analysis was performed using nonparametric tests, because the distribution of individual characteristics differed from a normal distribution (Shapiro-Wilk test). The following values were given in quantitative variables: mean, median, minimum, maximum, and standard deviation. The comparison of various quantitative characteristics in all patients was performed using the Mann-Whitney $\mathrm{U}$ test. The comparison of the different quantitative traits in separate groups was performed using the U Mann-Whitney test, Kruskal-Wallis test and multiple comparisons. The results were considered statistically significant when the calculated probability $\mathrm{p}<0.05$. The analysis was performed using Statistica 10.0 and Prism. 


\section{Results}

The patient histories did not reveal any signs and symptoms of masticatory system dysfunction. Given that the instrumental analysis considers separately the right and left temporomandibular joints, the data obtained for analysis were considered separately for the right and left joints. All patients underwent the examination of both temporomandibular joints representing the number of 374 temporomandibular joints (women-196, 52.4\% of the joints, men- 178, $47.6 \%$ of the joints). Open/Close Quantity Max S-3D in women and men varied significantly. The significance level for Mann-Whitney test was $\mathrm{p}=0.021$. Figure 1 shows the distribution of the values of the Quantity Max S-3d for each joint. The results of Open/Close Reproducibility 3 $\mathrm{mm}$ test in women and in men varied significantly. The significance level for the Mann-Whitney $U$ test was $p=0.022$. Figure 2 is a chart showing the distribution of the Open/Close Reproducibility $3 \mathrm{~mm}$ test for each joint. The results of the Open/Close Reproducibility $5 \mathrm{~mm}$ test in the men and the women also varied significantly. The significance level for the Mann-Whitney $U$ test was $p=0.022$. Figure 3 is a chart showing the distribution of the Open/Close Reproducibility $5 \mathrm{~mm}$ test for the joints in the men and the women.

Tables 1 and 2 show the parameters obtained from the computer analysis of the movements of abduction and adduction for the left and right temporomandibular joints.

The comparison of measurements obtained during the movement of adduction and abduction of the jaw between the right and left side allowed for finding statistically significant differences in the distribution of the Open/Close Sagittal Condyle Inclination Values $5 \mathrm{~mm}$.

Figure 4 is a graph illustrating Open/Close Sagittal Condyle Inclination Values $5 \mathrm{~mm}$ on the right and left side.

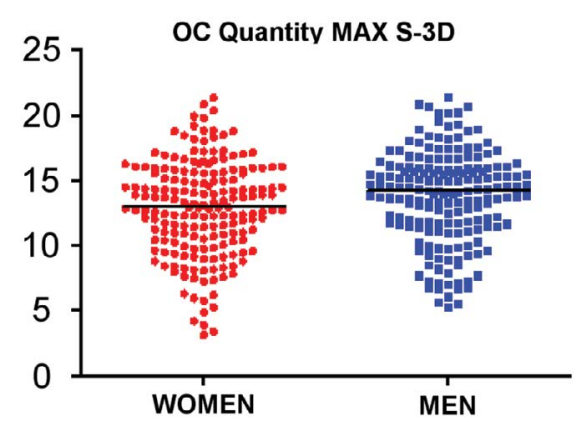

Figure 1: The distribution of the Quantity Max S-3D for the joints of the men and the women with a marked median value $(p=0.021)$.

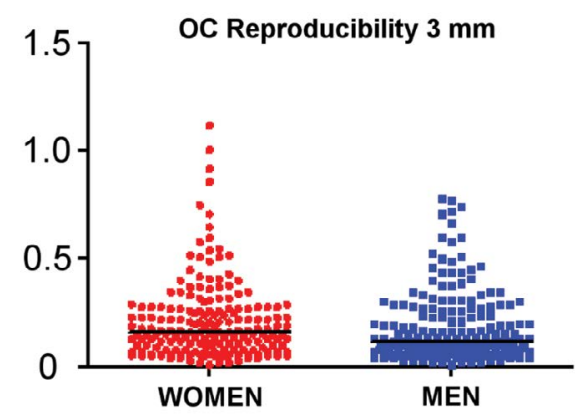

Figure 2: The distribution of the OC Reproducibility $3 \mathrm{~mm}$ test for the joints in the men and the women with a marked median value $(p=0.022)$

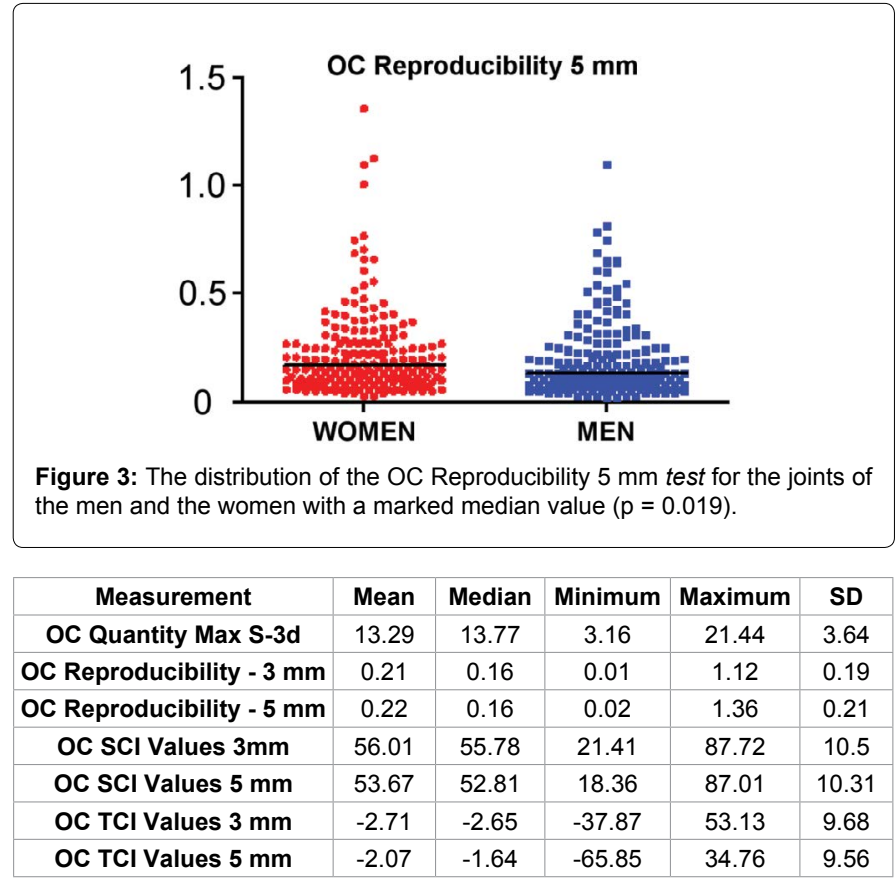

Table 1: Numerical characteristics of the measurements made by the device Cadiax Compact II for all of the left temporomandibular joints.

\begin{tabular}{|c|c|c|c|c|c|}
\hline Measurement & Mean & Median & Minimum & Maximum & $\begin{array}{c}\text { Standard } \\
\text { deviation }\end{array}$ \\
\hline $\begin{array}{c}\text { OC Quantity Max } \\
\text { S-3d }\end{array}$ & 13.32 & 13.7 & 3.45 & 21.4 & 3.64 \\
\hline $\begin{array}{c}\text { OC Reproducibility } \\
\text { 3mm }\end{array}$ & 0.19 & 0.13 & 0.02 & 0.92 & 0.16 \\
\hline $\begin{array}{c}\text { OC Reproducibility } \\
\text { 5mm }\end{array}$ & 0.21 & 0.15 & 0.02 & 1.1 & 0.18 \\
\hline OC SCI Values 3mm & 57.9 & 57.43 & 22.87 & 88.85 & 11.96 \\
\hline OC SCI Values 5 mm & 56.03 & 54.94 & 20.18 & 86.67 & 11.91 \\
\hline OC TCI Values 3 mm & -3.28 & -3.59 & -56.59 & 63.09 & 12.36 \\
\hline OC TCI Values 5 mm & -2.36 & -2.65 & -43.11 & 51.21 & 10.03 \\
\hline
\end{tabular}

OC-open/close, SCl- Sagittal Condyle Inclination, TCI - Transverse Condyle Inclination

Table 2: Numerical characteristics of the measurements made by the device Cadiax Compact II for all of the right temporomandibular joints.

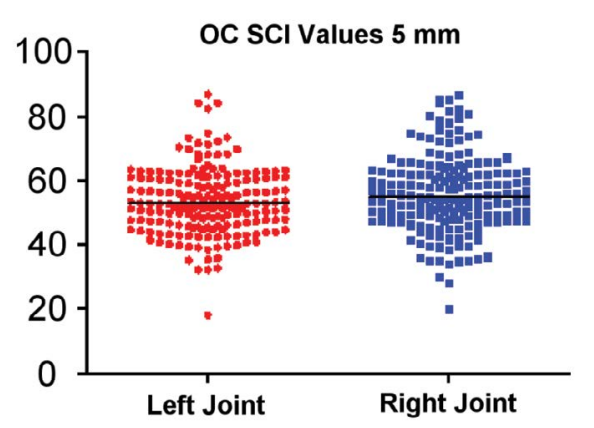

Figure 4: The distribution of $\mathrm{OC} \mathrm{SCI}$ Values $5 \mathrm{~mm}$ for the right and left joint with a marked median value $(p=0.04)$.

\section{Discussion}

According to the design of the study, examinations were performed on a group of generally healthy people, with full dental arches and normal appearing occlusal conditions and without any complaints 
related to their stomatognathic systems. The inclusion and exclusion criteria were defined so that, as much as possible, they excluded the influence of local factors (a lack of teeth, malocclusion, etc.) and any systemic effects on the function of the stomatognathic system.

Computed axiography is a good and looks to be reliable method for the diagnosis of diseases of the temporomandibular joints [26,27,29]. This fact was the main reason for choosing the research methods presented. All of the joints qualified for this study were recorded and analyzed using a device, Cadiax Compact II, which gives graphic records of opening and closing movements and their numeric values. Cadiax Compact II recorded and calculated OC Quantity Max S-3D. OC Quantity Max S-3D in men and women differed significantly $(\mathrm{p}=0.021)$. Smaller values of OC Quantity Max S-3D occurred in women $(12.89 \pm 3.66 \mathrm{~mm})$ than in men $(13.73 \pm 3.57 \mathrm{~mm})$. It should be pointed out that there was a large interval between the minimum and maximum values, which explains the large standard deviation. A large standard deviation indicates a wide span of numerical results. Considering the large differences in the OC Quantity Max S-3D in healthy people, this parameter cannot be the sole criterion for verifying proper TMJ function. The literature provides a different value for the OC Quantity Max S-3D in healthy patients. These values vary depending on the choice of the kinematic or arbitrary hinge axis as a point, which marks the beginning of the movement. In this study all plots of the mandibular movements were recorded from an arbitrary hinge axis, since it is the most common in dentistry due to its ease of use and the reproducibility of the registration determination [24]. In the case of devices for determining the terminal hinge axis, smaller values are usually recorded [22-24]. Using such devices Slavícek found that the normal range of the condylar movement should be $14 \mathrm{~mm}$ [22]. The similar apparatus was used by Travers et al. [23] who registered the value of $13.95 \mathrm{~mm}$ in the right joint and $14.50 \mathrm{~mm}$ in the left joint. Similar values were also recorded by Chen et al. [37] who studied the movements of the jaw using an optoelectronic device based on the arbitrary hinge axis. From a group of 25 healthy women and men they recorded the range of the condylar movement at $14.16 \mathrm{~mm}$. All of these values were similar to those obtained in this study. Yatabe et al. [24] investigated the use of the kinematic hinge axis and achieved the much larger values of $23.2 \pm 4.2 \mathrm{~mm}$ for the right TMJ and $23.1 \pm 4.5 \mathrm{~mm}$ for the left TMJ. In other studies Bernhardt et al. [28] compared the results recorded by the equipment measuring the movements of the mandible with the use of cameras, based on the kinematic terminal hinge axis, and reported similar to Yatabe values of the parameters [24].

Based on the results, we can conclude that women have a smaller range of condylar movement than men, which directly affects the smaller range of abduction of the mandible. It may be caused by the differences in the body and facial skeleton sizes between men and women [16]. The studies of numerous authors indicate that the range of motion is also affected by the height $[17,18]$ and the weight of the patient [19] which are both typically smaller in women. The smaller range of mandibular abduction and maximum condylar movement of the mandible in women were also reported in studies from other authors. Tipton noted significant differences in the length of the articular condyle path between males and females [20]. Studies carried out in Turkey among healthy subjects with Angle's Class I occlusion also showed a difference in the condylar range of movement between men and women [21]. Statistically significant differences in OC Quantity Max S-3D in women and men have also been noted in the studies conducted by Lewis, Buschang and Throckmorton [32]. Their tests were carried out using the optoelectronic pantomography in patients without locomotor masticatory system dysfunctions. In men, there was a range of condylar movement from $15.4 \mathrm{~mm}$ to $17.6 \mathrm{~mm}$, whereas in women, these values were lower and ranged from $12.4 \mathrm{~mm}$ to $12.7 \mathrm{~mm}$. Similar results were obtained in the group of women in this study $(12.89 \mathrm{~mm})$. Very similar results were registered by Fukui et al. [33] who studied 21 healthy women aged between 20 and 24 years using an optoelectronic device. Their OC Quantity Max S-3D was $12.8 \mathrm{~mm}$ [33]. A similar device was used to study another group of 25 healthy subjects, and the mean condylar movement was 14.16 $\mathrm{mm}$ for both sexes. In other studies, there were no differences in terms of the condylar movement during the opening movement in women and men. Kraljevic et al. [26] in a study of the OC Quantity Max S-3D using the Cadiax on healthy men and women did not find a statistically significant difference but the study was conducted on the group of 43 subjects. The mean OC Quantity Max S-3d during the opening in healthy individuals was $14.63 \mathrm{~mm}, 14.88 \mathrm{~mm}$ in men, and $14.41 \mathrm{~mm}$ in women. The studies of Turkish scientists using the Cadiax system showed that the graphs displaying the condylar movements showed slightly lower values in patients with TMJ dysfunction and were also characterized by a lower reproducibility of the movements $[34,35]$.

The examination carried out using the camera Cadiax Compact II recorded a lower Reproducibility of the opening or closing in women compared to men. Differences between the parameters were statistically significant $(\mathrm{p}=0.022)$. Greater Variability of the movements in women may be associated with Generalized Joint Laxity (GJL). The condition is more common in women and decreases with age. This is one theoretical explanation for the existence of a greater susceptibility to dysfunction in the female TMJ [5,6]. Generalized Joint Laxity is characterized by an increased range of motion in the joints, more than in the generally accepted standards [4,7], which allows hypermobility of the joint. Generalized Joint Laxity is combined with many TM] diseases. They concern subluxation, without associated symptoms of pain or dysfunction and the increased mobility of the articular disc [8,9] However, one study found no significant difference in TMJ mobility between control subjects and osteoarthrosis/internal derangement patients [10], indicating that the interaction may not be strong. De Coster et al. [11] concluded a positive relationship between GHJ and TMD, as did Deodato et al. [12], Hirsch et al. [13] evaluating 895 subjects 20 to 60 years of age found a significant relationship specifically between GHJ and likelihood of reducing disc displacements of the TMJ. Perrini et al. [14] also found a significant positive relationship between GJH and TMJ symptoms, while Greenwood did not [15].

Cadiax Compact II also registered the parameter of the Sagittal Condyle Inclination during the movement of abduction and adduction. This parameter in the test of $5 \mathrm{~mm}$ (OC SCI Values 5 $\mathrm{mm}$ ) showed statistically significant differences between the right and left side $(\mathrm{p}=0.04)$. OC SCI Values $5 \mathrm{~mm}$ is determined as the angle measured from the reference position with respect to the Frankfort plane. Statistical differences between the right and left OC SCI Values $5 \mathrm{~mm}$ may indicate an asymmetrical arrangement of the articular heads of the condyle, the posterior slopes of the eminences or an asymmetry in the facial structure [36]. The asymmetry of movement can be associated with the asymmetric arrangement of the heads of the condyles, which changes the measured OC Values SCI. The literature contains reports that the symmetry of the condylar heads in both TMJs suggests normal joint function [37,38], while other authors have observed asymmetric positions of the condylar heads in diseased locomotor masticatory systems [39,40]. Kenyworth et al. [40] who studied the path of movement of the mandible in healthy patients and those with locomotor masticatory system dysfunction, showed that in $63 \%$ of healthy subjects and in $100 \%$ of patients with the dysfunction, there was asymmetry of condylar movement. The asymmetry of the 
masticatory system can directly cause asymmetry of the movements taking place in the TMJs and therefore, asymmetry by itself, cannot constitute a criterion for determining the existence of locomotor masticatory system dysfunction.

The measurements of OC SCI Values $5 \mathrm{~mm}$ were similar for women and men (57.74 and 56.16 degrees, respectively). At the opening of $5 \mathrm{~mm}$, this angle was 54.94 for women, while in men 54.76 (the difference was 0.22 degree). The studies of other authors using axiography showed that the mean value of the SCI angle, measured in healthy individuals with Angle's Class I occlusions during opening and closing movements, was 69.6 in women whereas in men 72.7 degrees [21]. These values differ from those obtained in this study and may be associated with the use of a different recording device. The Sagittal Condyle Inclination seems to be a parameter which is consistent within and unique to each patient. Similar observations were made by Schierz et al. [35] in a group of patients aged $64 \pm 10.3$ years, where the SCI was investigated using Cadiax Compact, noting that this parameter is not constant and changes with the condition of the dentition.

\section{Conclusions}

The study results allowed us to formulate the following conclusions:

1. The graphs of opening and closing movements in a group of healthy women and men with no symptoms from the masticatory system differed significantly between genders.

2. The Quantity Maximal S3d parameter is higher in men, but because of the large range of values, this parameter cannot be a single criterion used to detect TMJ dysfunction.

3. The Reproducibility of condylar movement is not as consistent in women, which may indicate on average slightly greater generalized joint hypermobility.

4. The supplementary tests using Cadiax Compact II provide objective measurements contributory to the evaluation of the quality of function in the temporomandibular joints.

The use of modern axiographic device provides a simple and noninvasive way to detect small abnormalities in the motor function of the masticatory system enabling early preventive and therapeutic measures, which are of great importance in efficient dental treatment.

\section{References}

1. McNeill C (1997) Management of temporomandibular disorders: Concepts and controversies. J Prosthet Dent 77: 510-522.

2. Hassel AJ, Rammelsberg $P$, Schmitter $M(2006)$ Inter-examiner reliability in the clinical examination of temporomandibular disorders: influence of age. Community Dent Oral Epidemiol 34: 41-46.

3. Hirsch C, John MT, Lautenschlager C, List T (2006) Mandibular jaw movement capacity in 10-17-yr-old children and adolescents: normative values and the influence of gender age and temporomandibular disorders. Eur J Oral Sci 114 $465-470$.

4. Yazgan P, Geyikli I, Zeyrek D (2008) Is joint hypermobility important in prepubertal children? Rheumatol Int. 28: 445-451.

5. Russek LN (1999) Hypermobility syndrome. Phys Ther 79: 591-599.

6. Huddleston Slater JJ, Lobbezoo F, Onland-Moret NC, Naeije M (2007) Anterior disc displacement with reduction and symptomatic hypermobility in the human temporomandibular joint: prevalence rates and risk factors in children and teenagers. J Orofac Pain 21: 55-62.

7. Seçkin U, Tur BS, Yilmaz O, Yaqci I, Bodur H, et al. (2004) The prevalence of joint hypermobility among high school students. Rheumatol Int 25: 260-263.

8. Conti PC, Miranda JE, Araujo CR (2000) Relationship between systematic join laxity, TMJ hypertranslation and intra-articular disorders. Cranio 18: 192-197.
9. Lozano FJ (2009) Joint hypermobility and disk displacement confirmed by magnetic resonance imaging: A study of women with temporomandibula disorders. Oral Surg Oral Med Oral Pathol Oral Radiol Endod 107: e54-e57.

10. Dijkstra PU, de Bont LG, Stegenga B, Boering G (1992) Temporomandibular joint osteoarthrosis and generalized joint hypermobility. Cranio 10: 221-227.

11. De Coster PJ, Van den Berghe LI, Martens LC (2005) Generalized joint hypermobility and temporomandibular disorders: inherited connective tissue disease as a model with maximum expression. J Orofac Pain 19: 47-57.

12. Deodato F, Trusendi R, Giorgetti R, Scalese MU (2006) Predisposition for temporomandibular joint disorders: loose ligaments. Cranio 24: 179-183.

13. Hirsch C, John MT, Stang A (2008) Association between generalized join hypermobility and signs and diagnoses of temporomandibular disorders. Eur J Oral Sci 116: 525-530.

14. Perrini F, Tallents RH, Katzberg RW, Ribeiro RF, Kyrkanides S, et al. (1997) Generalized joint laxity and temporomandibular disorders. J Orafac Pain 11 215-221.

15. Greenwood LF (1987) Is temporomandibular joint dysfunction associated with generalized joint hypermobility? J Prosthet Dent 58: 701-703.

16. Dijkstra PU, Hof AL, Stegenga B, de Bont LG (1999) Influence of mandibular length on mouth opening. J Oral Rehabil 26: 117-122.

17. Landtwig K (1978) Evaluation of the normal range of vertical mandibula opening in children and adolescents with special reference to age and stature. J Maxillofac Surg 6: 157-162.

18. Vanderas AP (1992) Mandibular movements and their relationship to age and body height in children with or without clinical signs of craniomandibular dysfunction: Part IV. A comparative study. ASDC J Dent Child 59: 338-341.

19. Abou-Atme YS, Chedid N, Melis M, Zawawi KH (2008) Clinical measurement of normal maximum mouth opening in children. Cranio 26: 191-196.

20. Tipton NJ (1996) Normal masticatory function of children and young adults part II: range of motion. Thesis, Baylor College of Dentistry, Dallas.

21. Balos Tuncer B, Ozoğul B, Akkaya S (2011) Differences in opening and protrusive mandibular movements between Class I and II malocclusions in healthy adolescents. Korean J Orthod 41: 127-137.

22. Slavicek $R$ (1988) Clinical and instrumental functional analysis for diagnosis and treatment planning, Part 5.Axiography. J Clin Orthod 22: 656-667.

23. Traversa $\mathrm{KH}$, Buschanga $\mathrm{PH}$, Hayasakib H, Throckmorton GS (2000) Associations between incisor and mandibular condylar movements during maximum mouth opening in humans. Arch Oral Biol 45: 267-275.

24. Yatabe M, Zwijnenburg A, Megens CCEJ, Naeije M (1995) The kinematic center: a reference for condylar movements. J Dent Res 74: 1644-1648.

25. Tamaki K, Celar AG, Beyrer S, Aoki H (1997) Reproduction of exercusive tooth contact in a articulator with computerized axiography data. J Prosthet Dent 78 : 373-378.

26. Kraljevic S, Panduric J, Badel T, Dulcic N (2003) Electronic Axiography Coll Antropol 27: 51-59.

27. Piehslinger E, Celar RM, Horejs T, Slavicek R (1993) Orthopedic jaw movement observations. Part II: The rotational capacity of the mandible. Cranio 11: 206-210.

28. Bernhardt O, Küppers N, Rosin M, Meyer G (2003) Comparative tests of arbitrary and kinematic transverse horizontal axis recordings of mandibular movements. J Prosthet Dent 89: 175-179.

29. Lückerath W (1989) Comparison of mechanical and three-dimensional electronic axiography. Dtsch Zahnarztl Z 44: 754-757.

30. Dworkin SF, LeResche L (1992) Research diagnostic criteria fo temporomandibular disorders: criteria, examinations and specifications, critique. J Craniomandib Disord 6: 301-355.

31. Schiffman E, Ohrbach R, Truelove E, Look J, Anderson G, et al. (2014 Diagnostic Criteria for Temporomandibular Disorders (DC/TMD) for Clinical and Research Applications: recommendations of the International RDC/TMD Consortium Network and Orofacial Pain Special Interest Group. J Oral Facial Pain Headache 28: 6-27.

32. Lewis RP, Buschang PH, Throckmorton GS (2001) Sex differences in 
Citation: Kondrat W, Sierpinska T, Radke J (2018) Axiographic Assessment of Masticatory System function in Healthy Volunteers. Dentistry 8: 504. doi:10.4172/2161-1122.1000504

Page 6 of 6

mandibular movements during opening and closing. Am J Orhod Dentofacial Orthop 120: 294-303.

33. Fukui T, Tsuruta M, Murata K, Wakimoto Y, Tokiwa H, et al. (2002) Correlation between facial morphology, mouth opening ability, and condylar movement during opening-closing jaw movements in female adults with normal occlusion. Eur J Orthod 24: 327-336.

34. Akar GC, Erdem A, Ada E, Köse T (2008) Comparison of Clinical, Instrumental and Imaging Methods in Diagnosis of Temporomandibular Disorders. Acta Stomatol Croat 423: 242-254

35. Schierz O, Klinger N, Schön G, Reissmann DR (2014) The reliability of computerized condylar path angle assessment. Int J Comput Dent 17: 35-51.

36. Pirttiniemi P, Kantomaa T (1992) Relation of glenoid fossa morphology to mandibulofacial asymmetry, studied in dry human Lapp skulls. Acta Odontol Scand 50: 235-243.

37. Chen Q (1997) Relationship between craniofacial morphology and mandibular movement. Journal of the Osaka Odontological Society 60: 195-204.

38. Cohlmia JT, Ghosh J, Sinha PK, Nanda RS, Currier GF (1996) Tomographic assessment of temporomandibular joints patients with malocclusion. Angle Orthod 66: 27-35.

39. Katzberg RW, Westesson PL, Tallents RH, Drake CM (1996) Anatomic disorders of the temporomandibular joint disc in asymptomatic subjects. $\mathrm{J}$ Oral Maxillofac Surg 54: 147-152.

40. Kenyworth CR, Morrish RB Jr, Mohn C, Miller A, Swenson KA, et al. (1997) Bilateral condylar movement patterns in adult subjects. J Orofac Pain 11: 328-336. 